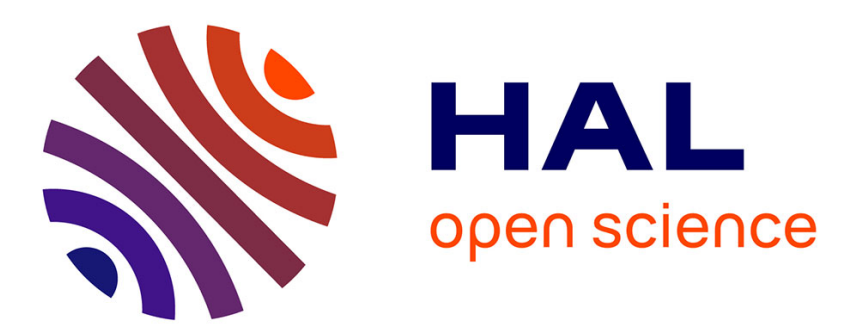

\title{
Jump conditions for unsteady small perturbations at fluid-solid interfaces in the presence of initial flow and prestress
}

Fabien Treyssede, Mabrouk Ben Tahar

\section{- To cite this version:}

Fabien Treyssede, Mabrouk Ben Tahar. Jump conditions for unsteady small perturbations at fluidsolid interfaces in the presence of initial flow and prestress. Wave Motion, 2009, 46 (2), pp 155-167. hal-00612172

\section{HAL Id: hal-00612172 \\ https://hal.science/hal-00612172}

Submitted on 28 Jul 2011

HAL is a multi-disciplinary open access archive for the deposit and dissemination of scientific research documents, whether they are published or not. The documents may come from teaching and research institutions in France or abroad, or from public or private research centers.
L'archive ouverte pluridisciplinaire HAL, est destinée au dépôt et à la diffusion de documents scientifiques de niveau recherche, publiés ou non, émanant des établissements d'enseignement et de recherche français ou étrangers, des laboratoires publics ou privés. 


\title{
Jump conditions for unsteady small perturbations at fluid-solid interfaces in the presence of initial flow and prestress
}

\author{
Fabien Treyssède* \\ Nantes Atlantique University, Laboratoire Central des Ponts et Chaussées, BP \\ 4129, 44341 Bouguenais, France \\ Mabrouk Ben Tahar
}

UTC, Laboratoire Roberval, BP 20529, 60205 Compiègne, France

\begin{abstract}
The goal of this paper is to investigate jump conditions for unsteady small perturbations at impermeable interfaces, slip or bonded, plane or not, between fluids and structures in the presence of initial flow and prestress. Based on conservative equations obtained from a mixed Eulerian-Lagrangian description, interface conditions are first derived in an elegant and straightforward manner thanks to the concept of generalized functions in distribution theory. These conditions are validated with exact conditions derived from a direct linearization of the standard jump conditions. For a straightforward comparison between both approaches, all conditions are written in terms of a curvilinear coordinate system attached to the interface. The normal Lagrangian displacement continuity across the interface is proved to be a sufficient condition. The jump conditions for mass, momentum, energy and entropy are discussed, yielding conditions for the Lagrangian perturbations of displacement, stress, heat flux and temperature. Displacement and stress jump conditions are shown to coincide with literature results. The mixed Eulerian-Lagrangian description is likely to be advantageous over its fully Eulerian or Lagrangian counterparts. It yields an interesting unification between existing formulations for inviscid fluids (Galbrun's equation) and solids (updated Lagrangian formulation), together with simpler jump conditions.
\end{abstract}

Key words: jump conditions, interface, vibration, acoustic, prestress, flow

* Corresponding author.

Email addresses: fabien.treyssede@lcpc.fr (Fabien Treyssède), mabrouk. bentahar@utc.fr (Mabrouk Ben Tahar). 


\section{Introduction}

Many practical applications involve small wave motion through an interface between initially moving fluids and/or prestressed solids. The context of this paper is restricted to impermeable interfaces (immiscible media always in contact with no void). Examples of fluid-solid interaction problems are numerous: wave propagation inside prestressed flow ducts (aeroengine ducts, human arteries), fluid-filled pressurized boreholes (acoustic measurements in geophysics) and immersed prestressed materials (ultrasonic stress characterization); dynamics and stability of pipes conveying fluid (turbomachinery components, heat exchangers, jet pumps, nuclear reactors,...) and fluid-filled cavities (storage tanks, human eyes,...). Some typical solid-solid and fluid-fluid problems can be encountered in the analysis of wave propagation inside composite materials (for stress measurements) and inside stratified flows under gravity (ocean). As examples, the reader could refer to [1-8] for papers dealing with some of the above mentioned applications. Another application of interest could also deal with the sensitivity analysis for the design and control of coupled fluid-structure systems $[9,10]$.

The motion of particles at the interface between both media in contact can occur with (resp. without) slip, as it is the case of inviscid (resp. viscous) fluid-structure interactions for example. In the absence of flow and prestress, interface conditions are well-established and the kinematic and dynamic jump conditions respectively correspond to the continuity of the normal components of the acoustic velocity or displacement (as well as their tangential components in the no slip case) and of the acoustic stress tensor. However, the derivation of the appropriate interface conditions is somewhat complicated when an initial flowing or prestressed state exists. Besides, the possibility of slip further complicates the derivation of jump conditions because material particles that are in contact at the interface in the current (Eulerian) configuration are no longer adjacent in another reference configuration, so that surface elements do not transform in the same manner on either side of the interface.

As far as the acoustic kinematic jump condition is concerned for an inviscid flowing fluid, considerable discussion appeared years ago in the literature as to whether continuity of particle normal displacement or normal velocity is the appropriate boundary condition. Nayfeh et al. [11] reviewed theoretical, numerical and experimental studies of the subject and concluded that the appropriate boundary condition was the continuity of particle normal displacement instead of the normal velocity. Myers [12] derived a kinematic condition based on the Eulerian acoustic velocity, valid at any impermeable vibrating surface that is stationary in its unperturbed configuration. This derivation had been later simplified by Farassat and Dunn [13]. Poirée [14,15] proposed using the continuity of normal Lagrangian (particle) displacement but without formal 
general proof. Godin $[16,17]$ finally proved the equivalence between the latter and Myers condition.

Few papers deal with the dynamic jump condition for a slip interface in the presence of prestress. Based on the assumption of the continuity of normal particle displacement, Poirée $[14,15]$ used distribution theory in order to derive a stress jump condition for plane interfaces and Goy [18] later extended his work to arbitrary non-plane interfaces (without accounting for geometric compatibility conditions). However, no general validation of their theoretical results were provided. Godin $[16,17]$ derived some exact linearized boundary conditions on the interface between two moving inviscid fluids. When studying incompressible hydroelastic vibrations, Schotté and Ohayon [19] obtained linearized boundary conditions on the interface between a prestressed structure and an inviscid fluid in the absence of flow. Norris et al. [20] made a thorough analysis of non-moving fluid/solid composite systems. Under the implicit assumption of homogeneous prestress along the interface, they derived some stress jump conditions valid for both slip and bonded interfaces. It should be noticed that most of these studies point out the benefits of an intermediate formulation compared to a full Lagrangian or Eulerian approach. This intermediate formulation consists in writing the equilibium equations with respect to the unperturbed coordinates corresponding to the prestress state. This intermediate formulation is often referred to as "updated Lagrangian" in non-linear mechanics and sometimes "mixed Eulerian-Lagrangian" in flow acoustics.

The goal of this paper is to investigate and clarify jump conditions that hold for linear perturbations at any impermeable interfaces (slip or bonded, plane or not) between fluids and/or structures in the presence of initial flow and prestress. Based on a mixed Eulerian-Lagrangian formulation (recalled in Sec. 2) together with the concept of generalized functions in distribution theory [2123], interface conditions associated with general conservative equations are derived in Sec. 3. These conditions generalize Poirée's results [14,15] to nonplane interfaces. The approach chosen is the same as in Refs. $[14,15,18]$ and allows an elegant and rather straightforward derivation. In Sec. 4, interface conditions for perturbations are validated through a comparison with some exact conditions derived from a linearization of the standard Eulerian jump conditions. For a straightforward comparison, conditions based on both approaches are written in terms of a curvilinear coordinate system attached to the interface. In particular, we prove the sufficiency of normal displacement continuity across the interface (without requiring its initial stationarity). In Sec. 5, jump conditions for mass, momentum, energy and entropy are explicitly given. The kinematic and dynamic jump conditions are discussed and shown to coincide with standard literature results. Section 6 finally concludes this paper. 


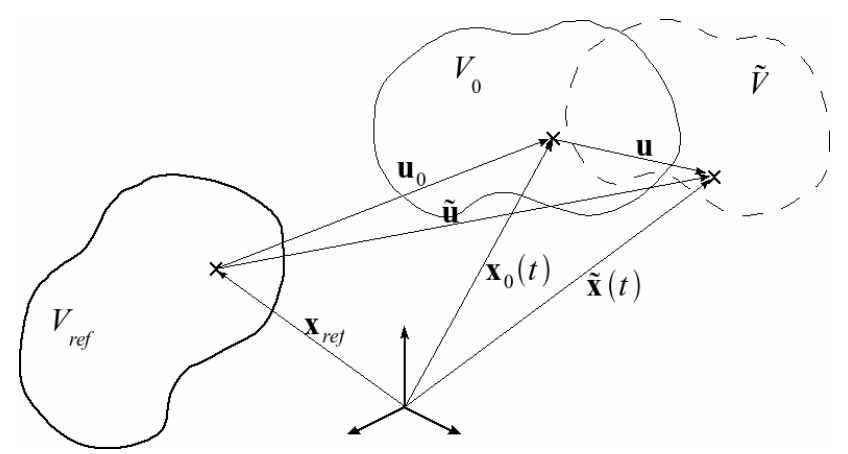

Fig. 1. Reference (bold line), intermediate (thin), and current (dashed) configurations with their respective coordinates $\mathbf{x}_{r e f}, \mathbf{x}_{0}(t)$ and $\tilde{\mathbf{x}}(t)$, denoting the particle position. $\mathbf{u}_{0}$ and $\tilde{\mathbf{u}}$ are respectively the intermediate and current displacements, $\mathbf{u}$ is the displacement perturbation.

\section{Mixed Eulerian-Lagrangian description}

As shown in Fig. 1, three configurations can be distinguished in the linear dynamic analysis of continuum media that are not initially at rest: the reference configuration (corresponding to a state of complete rest, quiescent and unprestressed), the intermediate configuration (unperturbed, corresponding to the flowing/prestressed state without small oscillatory perturbations), and the current (Eulerian) configuration including some small superimposed oscillatory perturbations. Physical fields referring to these configurations will respectively be denoted with a subscript ref, a subscript 0 and a tilde. The absence of symbol will be left for superimposed oscillatory perturbations. The material position vector in each configuration will be denoted $\mathbf{x}_{r e f}, \mathbf{x}_{0}(t)$ and $\tilde{\mathbf{x}}(t)$ - corresponding to the Lagrangian, intermediate and Eulerian coordinates - and the gradients with respect to them will be denoted by $\nabla_{\text {ref }}, \nabla_{0}$ and $\tilde{\nabla}$. For simplicity, the same notation will be kept for physical fields written in terms of any of the above mentioned coordinates (this is source of possible confusion but the reader can easily discern the coordinates used by context). Table 1 summarizes the different notations for the three configurations.

For the sake of clarity and self-containedness, this section mainly reviews the mixed Eulerian-Lagrangian description and the associated governing equilibrium equations though it has been presented elsewhere - see for instance Ref. [15]. Section 2.5 outlines how such a description indeed provides an interesting unification between existing solid and fluid formulations.

\subsection{Current and intermediate equilibrium states}

In fluid and solid continuum mechanics, a vector equilibrium equation describing the current state (occupying a volume $\tilde{V}$ ) by means of the current 
Table 1

Notations in the three configurations.

\begin{tabular}{ccccc}
\hline $\begin{array}{c}\text { Configurations } \\
\text { (state) }\end{array}$ & $\begin{array}{c}\text { Position } \\
\text { (coordinates) }\end{array}$ & $\begin{array}{c}\text { Volume, } \\
\text { interface }\end{array}$ & $\begin{array}{c}\text { Density, } \\
\text { temperature }\end{array}$ & $\begin{array}{c}\text { Displ., velocity, } \\
\text { Cauchy stress,... }\end{array}$ \\
\hline \hline Reference & $\mathbf{x}_{\text {ref }}$ & $V_{\text {ref }}$ & $\rho_{\text {ref }}$ & $\mathbf{0}, \mathbf{0}$ \\
$($ rest $)$ & $($ Lagrangian) & $\Sigma_{r e f}, \mathbf{n}_{r e f}$ & $T_{r e f}$ & $\mathbf{0}, 0,0,0$ \\
\hline Intermediate & $\mathbf{x}_{0}$ & $V_{0}$ & $\rho_{0}$ & $\mathbf{u}_{0}=\mathbf{x}_{0}-\mathbf{x}_{r e f}, \mathbf{v}_{0}$ \\
(prestressed) & $($ intermediate) & $\Sigma_{0}, \mathbf{n}_{0}$ & $T_{0}$ & $\boldsymbol{\sigma}_{0}, e_{0}, q_{0}, s_{0}$ \\
\hline Current & $\tilde{\mathbf{x}}$ & $\tilde{V}$ & $\tilde{\rho}$ & $\tilde{\mathbf{u}}=\tilde{\mathbf{x}}-\mathbf{x}_{r e f}, \tilde{\mathbf{v}}$ \\
$($ perturbed) & $($ Eulerian $)$ & $\tilde{\Sigma}, \tilde{\mathbf{n}}$ & $\tilde{T}$ & $\tilde{\boldsymbol{\sigma}}, \tilde{e}, \tilde{q}, \tilde{s}$ \\
\hline
\end{tabular}

(Eulerian) coordinates has the following general Eulerian conservative form:

$$
\frac{\partial(\tilde{\rho} \tilde{\mathbf{a}})}{\partial t}+\tilde{\nabla} \cdot(\tilde{\rho} \tilde{\mathbf{a}} \otimes \tilde{\mathbf{v}}+\tilde{\mathbf{A}})=\tilde{\rho} \tilde{\boldsymbol{\alpha}} \text { in } \tilde{V}
$$

$\tilde{\rho}$ and $\tilde{\mathbf{v}}$ are respectively the density and the velocity vector. $\tilde{\mathbf{a}}, \tilde{\boldsymbol{\alpha}}$ denote vectors and $\tilde{\mathbf{A}}$ denotes a second order tensor with appropriate units. A scalar equilibrium equation has the following general conservative form:

$$
\frac{\partial(\tilde{\rho} \tilde{b})}{\partial t}+\tilde{\nabla} \cdot(\tilde{\rho} \tilde{b} \tilde{\mathbf{v}}+\tilde{\mathbf{b}})=\tilde{\rho} \tilde{\beta} \text { in } \tilde{V}
$$

where $\tilde{b}, \tilde{\beta}$ are scalars and $\tilde{\mathbf{b}}$ is a vector.

The balance equations for mass, momentum, energy and entropy are obtained with the following identifications:

$$
\begin{array}{ll}
\text { mass: } & \tilde{b}=1, \tilde{\mathbf{b}}=\mathbf{0}, \tilde{\beta}=0 \\
\text { momentum: } & \tilde{\mathbf{a}}=\tilde{\mathbf{v}}, \tilde{\mathbf{A}}=-\tilde{\boldsymbol{\sigma}}, \tilde{\boldsymbol{\alpha}}=\tilde{\mathbf{f}} \\
\text { energy: } & \tilde{b}=\tilde{e}+1 / 2 \tilde{\mathbf{v}} \cdot \tilde{\mathbf{v}}, \tilde{\mathbf{b}}=\tilde{\mathbf{q}}-\tilde{\boldsymbol{\sigma}} \cdot \tilde{\mathbf{v}}, \tilde{\beta}=\tilde{\mathbf{f}} \cdot \tilde{\mathbf{v}}+\tilde{r} \\
\text { entropy: } & \tilde{b}=\tilde{s}, \tilde{\mathbf{b}}=\tilde{\mathbf{q}} / \tilde{T}, \tilde{\beta}=\tilde{r} / \tilde{T}
\end{array}
$$

where $\tilde{\boldsymbol{\sigma}}, \tilde{e}, \tilde{\mathbf{q}}, \tilde{s}$ and $\tilde{T}$ respectively denote the Cauchy stress tensor, the internal energy per unit mass, the heat flux, the entropy per unit mass and the temperature. $\mathbf{f}$ and $\tilde{r}$ are the external body force and heat source per unit mass. Replacing tildes with subscripts 0, Eqs. (1)-(3) also hold for the intermediate configuration state. Note that for irreversible process, the equality in Eq. (2) written for entropy should be replaced with the inequality $\geq$. 


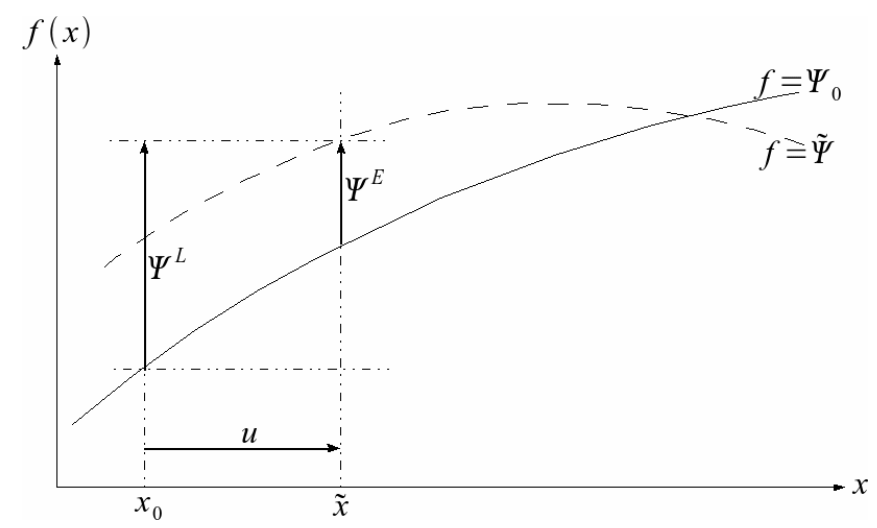

Fig. 2. For the one-dimensional case, plots of intermediate field $\Psi_{0}(x)$ (thin line) and current field $\tilde{\Psi}(x)$ (dashed) at a fixed time. $x_{0}$ and $\tilde{x}$ are respectively the particle position in the intermediate and current states, $u=\tilde{x}-x_{0}$ being the (Lagrangian) perturbation of displacement. Then, $\Psi^{E}=\tilde{\Psi}(\tilde{x})-\Psi_{0}(\tilde{x})$ and $\Psi^{L}=\tilde{\Psi}(\tilde{x})-\Psi_{0}\left(x_{0}\right)$ are respectively the graphical representations of the Eulerian and Lagrangian perturbations of $\tilde{\Psi}$.

\subsection{Definitions}

One can choose to write physical fields with respect to the Lagrangian $\left(\mathbf{x}_{r e f}, t\right)$, intermediate $\left(\mathbf{x}_{0}(t), t\right)$ or Eulerian $(\tilde{\mathbf{x}}(t), t)$ variables. Denoting $\mathbf{u}$ as the particle small oscillatory displacement, $\mathbf{x}_{0}$ and $\tilde{\mathbf{x}}$ are related by (see Fig. 1 ):

$$
\tilde{\mathbf{x}}=\mathbf{x}_{0}+\epsilon \mathbf{u}
$$

Let $\tilde{\Psi}(\tilde{\mathbf{x}}, t)$ denote any field - scalar, vector or tensor - describing the current state in terms of Eulerian coordinates. Both the following linear perturbations can be defined:

$$
\epsilon \Psi^{E}=\tilde{\Psi}(\tilde{\mathbf{x}}, t)-\Psi_{0}(\tilde{\mathbf{x}}, t), \epsilon \Psi^{L}=\tilde{\Psi}(\tilde{\mathbf{x}}, t)-\Psi_{0}\left(\mathbf{x}_{0}, t\right)
$$

Superscripts $E$ and $L$ respectively denote Eulerian and Lagrangian perturbations (one exception being for the displacement perturbation $\mathbf{u}=\mathbf{u}^{L}$ in order to avoid cumbersome expressions). Figure 2 exhibits a graphical representation of both perturbations in the one-dimensional case.

Often implicitly used in fluid acoustics, Eulerian perturbations are clearly associated with the same geometrical point but not necessarily the same particle. Lagrangian perturbations, usually used in solid mechanics, are associated to the same particle but here, the Lagrangian perturbations defined by Eq. (5) are written with respect to $\left(\mathbf{x}_{0}, t\right)$ (or equivalently $(\tilde{\mathbf{x}}, t)$ because of the assumption of small perturbations between the intermediate and the current configurations). This description is thus mixed and may be called mixed Eulerian-Lagrangian description in contrast with a full Lagrangian description, where Lagrangian perturbations would be written with respect to the 
Lagrangian variables $\left(\mathbf{x}_{r e f}, t\right)$.

From Eqs. (4) and (5), the following fundamental relation between Eulerian and Lagrangian first order perturbations holds:

$$
\Psi^{L}=\Psi^{E}+\left(\mathbf{u} \cdot \nabla_{0}\right) \Psi_{0}
$$

which shows that Eulerian and Lagrangian perturbations of $\tilde{\Psi}$ are equivalent only if $\Psi_{0}$ remains constant.

\subsection{Perturbation rules}

As for a full Lagrangian or Eulerian approach, the Lagrangian perturbations of sum and product with a mixed Eulerian-Lagrangian description are simply $(\tilde{\Psi}+\tilde{\Phi})^{L}=\Psi^{L}+\Phi^{L}$ and $(\tilde{\Psi} \tilde{\Phi})^{L}=\Psi_{0} \Phi^{L}+\Psi^{L} \Phi_{0}$. However, the perturbation of derivatives is not straightforward (differentiation and perturbation operations do not commute). To the first order, it can be shown that:

$$
(\tilde{\nabla} \tilde{\Psi})^{L}=\nabla_{0} \Psi^{L}-\nabla_{0} \Psi_{0} \cdot \nabla_{0} \mathbf{u},\left(\frac{\partial \tilde{\Psi}}{\partial t}\right)^{L}=\frac{\partial \Psi^{L}}{\partial t}-\nabla_{0} \Psi_{0} \cdot \frac{\partial \mathbf{u}}{\partial t}
$$

One consequence is that $(\tilde{d} \tilde{\Psi} / d t)^{L}=d_{0} \Psi^{L} / d t$, where $\tilde{d} / d t=\partial / \partial t+(\tilde{\mathbf{v}} \cdot \tilde{\nabla})$ and $d_{0} / d t=\partial / \partial t+\left(\mathbf{v}_{0} \cdot \nabla_{0}\right)$ are the material derivatives with respect to the current and intermediate configurations. In particular, $\mathbf{v}^{L}=d_{0} \mathbf{u} / d t$.

\subsection{Perturbation of the equilibrium equations}

Applying the perturbation rules (7) to the balance equation (2) for mass $(\{\tilde{b}, \tilde{\mathbf{b}}, \tilde{\beta}\}=\{1, \mathbf{0}, 0\})$ and taking into account the intermediate equilibrium state yields: $d_{0}\left(\rho^{L} / \rho_{0}+\nabla_{0} \cdot \mathbf{u}\right) / d t=0$. Under the assumption of no wave-flow synchronism $[16,17]$, integration of this equation gives:

$$
\frac{\rho^{L}}{\rho_{0}}+\nabla_{0} \cdot \mathbf{u}=0 \text { in } V_{0}
$$

Perturbation rules are then applied to the general conservative Eqs. (1) and (2). Thanks to Eq. (8), the intermediate state equilibrium and the identity $\nabla_{0}$. $\left(\left(\nabla_{0} \cdot \mathbf{u}\right) \mathbf{I}-\nabla_{0} \mathbf{u}^{T}\right)=\mathbf{0}$, it can be shown that the resulting perturbed equations can be rewritten as the following conservative forms:

$$
\frac{\partial\left(\rho_{0} \mathbf{a}^{L}\right)}{\partial t}+\nabla_{0} \cdot\left(\rho_{0} \mathbf{a}^{L} \otimes \mathbf{v}_{0}+\mathbf{A}^{L}+\mathbf{A}_{0} \cdot\left(\left(\nabla_{0} \cdot \mathbf{u}\right) \mathbf{I}-\nabla_{0} \mathbf{u}^{T}\right)\right)=\rho_{0} \boldsymbol{\alpha}^{L} \text { in } V_{0}
$$


and:

$$
\frac{\partial\left(\rho_{0} b^{L}\right)}{\partial t}+\nabla_{0} \cdot\left(\rho_{0} b^{L} \mathbf{v}_{0}+\mathbf{b}^{L}+\mathbf{b}_{0} \cdot\left(\left(\nabla_{0} \cdot \mathbf{u}\right) \mathbf{I}-\nabla_{0} \mathbf{u}^{T}\right)\right)=\rho_{0} \beta^{L} \text { in } V_{0}
$$

where $\mathbf{I}$ is the second order identity tensor. Though the derivation of conservative Eqs. (9) and (10) is not straightforward from perturbation rules (7), it should be mentioned that such conservative forms are directly obtained with a transformation from current to intermediate variables in Refs. [14,15].

For clarity, the balance equations for momentum, energy and entropy are written with the identifications:

$$
\begin{array}{ll}
\text { momentum: } & \mathbf{a}^{L}=\mathbf{v}^{L}, \mathbf{A}^{L}=-\boldsymbol{\sigma}^{L}, \boldsymbol{\alpha}^{L}=\mathbf{f}^{L}, \mathbf{A}_{0}=-\boldsymbol{\sigma}_{0} \\
\text { energy: } & b^{L}=e^{L}+\mathbf{v}_{0} \cdot \mathbf{v}^{L}, \mathbf{b}^{L}=\mathbf{q}^{L}-\boldsymbol{\sigma}_{0} \cdot \mathbf{v}^{L}-\boldsymbol{\sigma}^{L} \cdot \mathbf{v}_{0}, \\
& \beta^{L}=\mathbf{f}_{0} \cdot \mathbf{v}^{L}+\mathbf{f}^{L} \cdot \mathbf{v}_{0}+r^{L}, \mathbf{b}_{0}=\mathbf{q}_{0}-\boldsymbol{\sigma}_{0} \cdot \mathbf{v}_{0} \\
\text { entropy: } & b^{L}=s^{L}, \mathbf{b}^{L}=\mathbf{q}^{L} / T_{0}-\mathbf{q}_{0} T^{L} / T_{0}^{2}, \\
& \beta^{L}=r^{L} / T_{0}-r_{0} T^{L} / T_{0}^{2}, \mathbf{b}_{0}=\mathbf{q}_{0} / T_{0}
\end{array}
$$

obtained from the Lagrangian perturbation of (3).

\subsection{Note upon stress tensors usually defined in solid and fluid mechanics}

The Cauchy stress increment $\boldsymbol{\sigma}^{L}$ (Lagrangian perturbation of Cauchy stress, as defined by Eq. (5)), is barely used in solid mechanics [24-26]. However another kind of stress increment, denoted $\boldsymbol{\sigma}$ for simplicity, naturally appears from a transformation from current to intermediate coordinates: $\boldsymbol{\sigma}=\operatorname{det}\left(\tilde{\mathbf{X}}_{0}\right) \tilde{\mathbf{X}}_{0}^{-1} \tilde{\boldsymbol{\sigma}} \tilde{\mathbf{X}}_{0}^{-T}-$ $\boldsymbol{\sigma}_{0}$, where $\tilde{\mathbf{X}}_{0}=\nabla_{0} \tilde{\mathbf{x}}=\mathbf{I}+\nabla_{0} \mathbf{u}$ (the deformation gradient from the intermediate to the current configuration). In non-linear mechanics and acoustoelasticity, $\boldsymbol{\sigma}$ may be referred to as the updated Kirchhoff stress increment tensor in the so-called updated Lagrangian formulation - see Bathe [27], Yang and Kuo [28] or Pao et al. [29] for instance. The updated Lagrangian formulation and the mixed Eulerian-Lagrangian formulation are obviously equivalent for small incremental displacements. A formal proof is obtained by linearizing the previous definition of $\boldsymbol{\sigma}$, which yields the following relationship:

$$
\boldsymbol{\sigma}^{L}+\boldsymbol{\sigma}_{0} \cdot\left(\left(\nabla_{0} \cdot \mathbf{u}\right) \mathbf{I}-\nabla_{0} \mathbf{u}^{T}\right)=\boldsymbol{\sigma}+\nabla_{0} \mathbf{u} \cdot \boldsymbol{\sigma}_{0}
$$

Using Eq. (12) into Eq. (9) written for momentum (see identification (11)) and simplifying gives the following equation: $\rho_{0} d_{0}^{2} \mathbf{u} / d t^{2}-\nabla_{0} \cdot\left(\boldsymbol{\sigma}+\nabla_{0} \mathbf{u}\right.$. $\left.\boldsymbol{\sigma}_{0}\right)=\rho_{0} \mathbf{f}^{L}$, corresponding to the so-called linearized updated Lagrangian formulation. For a linearly elastic solid, the stress-strain relationship is simply given by $\boldsymbol{\sigma}=\mathbf{C}: \boldsymbol{\epsilon}$, where $\boldsymbol{\epsilon}=1 / 2\left(\nabla_{0} \mathbf{u}+\nabla_{0} \mathbf{u}^{T}\right)$ is the linearized incremental 
strain tensor and $\mathbf{C}$ is the constitutive tensor. Note that for large prestrains, this constitutive tensor cannot be approximated as the constitutive tensor obtained in a total Lagrangian formulation and generally depends upon the predisplacement field [27-29].

For inviscid fluids, $\tilde{\boldsymbol{\sigma}}=-\tilde{p} \mathbf{I}$ and $\boldsymbol{\sigma}^{L}=-p^{L} \mathbf{I}$. $p^{L}$ is barely used in acoustics also (Eulerian perturbations being usually preferred). Nevertheless, it should be outlined that the Eulerian-Lagrangian description yields an interesting equation describing wave propagation in arbitrary inviscid flowing fluids. Obtained using Eqs. (8) and (9) for momentum together with the state equation $p^{L}=c_{0}^{2} \rho^{L}$ ( $c_{0}$ is the intermediate sound celerity), this wave equation is only written in terms of $\mathbf{u}$ and has some interesting properties. It is sometimes called Galbrun's equation - for further details, see Refs. [14,15,30-32].

The use of Lagrangian perturbations written in terms of the intermediate coordinates hence allows a straightforward unification between existing fluid and solid formulations. Moreover, their interest lies in the fact that Eqs. (8)(10) can be interpreted in the sense of distributions, as shown in the next sections.

\section{Interface conditions}

\subsection{Interface conditions for the current and intermediate equilibrium states}

One elegant way to derive jump conditions is to interpret Eqs. (1)-(3) in terms of distribution theory through the concept of generalized derivatives and to postulate that these equations hold in the sense of distributions [15,21-23].

Let us denote $[\cdot]_{\tilde{\Sigma}}$ the jump $+(\cdot)-_{-}(\cdot)$ on the moving interface, denoted $\tilde{\Sigma}$. This interface is represented by the implicit equation $\widetilde{S}(\tilde{\mathbf{x}}, t)=0$. Left subscripts - and + denote the media considered, respectively defined by $\tilde{S}<0$ and $\tilde{S}>0$. The unit normal is denoted $\tilde{\mathbf{n}}(\tilde{\mathbf{x}}, t)$ and will be oriented from - medium to + , so that $\tilde{\mathbf{n}}=\tilde{\nabla} \tilde{S} /|\tilde{\nabla} \tilde{S}|$. The surface $\tilde{S}=0$ is assumed smooth $(\tilde{S}$ is differentiable).

Details of calculations are given in Appendix A for general conservative equations. Neglecting any surface phenomenon, the same decomposition as Eqs. (A.2) and (A.3) without Dirac terms $\left({ }_{\delta} \tilde{\mathbf{A}}=\mathbf{0}\right.$ and $\left.{ }_{\delta} \tilde{\mathbf{b}}=\mathbf{0}\right)$ is applied for all the physical fields explicitly involved in the conservative Eqs. (1) -(3). For immiscible media, the following interface conditions are then obtained:

$$
[\tilde{\mathbf{A}} \cdot \tilde{\mathbf{n}}]_{\tilde{\Sigma}}=\mathbf{0},[\tilde{\mathbf{b}} \cdot \tilde{\mathbf{n}}]_{\tilde{\Sigma}}=0
$$


Replacing tildes with subscripts 0, Eqs. (13) also hold for the intermediate configuration state.

The interface conditions associated with the mass, momentum, energy and entropy equations are directly obtained from Eqs. (13) with the identification (3), yielding:

$$
[\tilde{\mathbf{v}} \cdot \tilde{\mathbf{n}}]_{\tilde{\Sigma}}=0,[\tilde{\boldsymbol{\sigma}} \cdot \tilde{\mathbf{n}}]_{\tilde{\Sigma}}=\mathbf{0},[\tilde{\mathbf{q}} \cdot \tilde{\mathbf{n}}]_{\Sigma}=(\tilde{\boldsymbol{\sigma}} \cdot \tilde{\mathbf{n}}) \cdot[\tilde{\mathbf{v}}]_{\tilde{\Sigma}},[\tilde{\mathbf{q}} \cdot \tilde{\mathbf{n}} / \tilde{T}]_{\tilde{\Sigma}} \geq 0
$$

Usually obtained from a pillbox analysis, these conditions are well-known in continuum mechanics (see for instance Refs. [33,34]). This a posteriori proves the exactness of conditions (14) as well as the validity of Eqs. (1)-(3) in the sense of distributions. When the interface is bonded $\left([\tilde{\mathbf{v}}]_{\tilde{\Sigma}}=\mathbf{0}\right)$ or involves an inviscid fluid $(\tilde{\boldsymbol{\sigma}} \cdot \tilde{\mathbf{n}}=-\tilde{p} \tilde{\mathbf{n}})$, the third and fourth conditions simplify into $[\tilde{\mathbf{q}} \cdot \tilde{\mathbf{n}}]_{\Sigma}=0$ and $(\tilde{\mathbf{q}} \cdot \tilde{\mathbf{n}})[1 / \tilde{T}]_{\tilde{\Sigma}} \geq 0$.

\subsection{Interface conditions for perturbations}

Following the same procedure as previously, one postulates that Eqs. (8)-(10) are valid in the sense of distribution theory. All derivatives are then interpreted as generalized derivatives. In the intermediate configuration, the interface is now denoted $\Sigma_{0}$ and is represented by the implicit equation $S_{0}\left(\mathbf{x}_{0}, t\right)=0$. The general results of Appendix A allow a direct derivation of the interface conditions associated with Eqs. (8)-(10).

First, Eq. (8) can be considered as a scalar conservative equation identified from Eq. (A.8) with $\{b, \mathbf{b}, \rho \beta\}=\left\{0, \mathbf{u},-\rho^{L} / \rho_{0}\right\}$ (and $\nabla=\nabla_{0}$ ), so that the associated jump condition is simply:

$$
\left[\mathbf{u} \cdot \mathbf{n}_{0}\right]_{\Sigma_{0}}=0
$$

meaning that the normal Lagrangian perturbation of displacement should be continuous across the interface.

Eq. (9) is a vector conservative equation that can be identified from Eq. (A.1) with $\rho=\rho_{0}, \mathbf{v}=\mathbf{v}_{0}, \mathbf{a}=\mathbf{a}^{L}, \mathbf{A}=\mathbf{A}^{L}+\mathbf{A}_{0} \cdot\left(\left(\nabla_{0} \cdot \mathbf{u}\right) \mathbf{I}-\nabla_{0} \mathbf{u}^{T}\right)$ and $\boldsymbol{\alpha}=\boldsymbol{\alpha}^{L}$. Eq. (10) is a scalar conservative equation identified from Eq. (A.8) in a similar manner. Because of the spatial derivatives of $\mathbf{u}$, the decomposition of $\mathbf{A}$ and b includes Dirac terms, given by:

$$
{ }_{\delta} \mathbf{A}=\left\{\left|\nabla_{0} S_{0}\right|\left(\left(\mathbf{A}_{0} H\left(-S_{0}\right)+{ }_{+} \mathbf{A}_{0} H\left(+S_{0}\right)\right)\left[\mathbf{u} \cdot \mathbf{n}_{0}\right]-\left(\mathbf{A}_{0} \cdot \mathbf{n}_{0}\right) \otimes[\mathbf{u}]\right)\right\}_{\Sigma_{0}}
$$

together with a similar expression for ${ }_{\delta} \mathbf{b}$. The first term of the right member inside parenthesis would be undefined but vanishes thanks to Eq. (15). The second term is well-defined thanks to the continuity of $\mathbf{A}_{0} \cdot \mathbf{n}_{0}\left(\right.$ and $\mathbf{b}_{0} \cdot \mathbf{n}_{0}$ ) 
across the interface. Then, it must be noted that the conditions ${ }_{\delta} \mathbf{A} \cdot \mathbf{n}_{0}=\mathbf{0}$ and ${ }_{\delta} \mathbf{b} \cdot \mathbf{n}_{0}=0$ on $\Sigma_{0}$ of Appendix A are ensured thanks to the continuity condition (15). From Eqs. (A.10), the following interface conditions are finally obtained:

$$
\left[\left(\mathbf{A}^{L}+\mathbf{A}_{0} \cdot\left(\left(\nabla_{0} \cdot \mathbf{u}\right) \mathbf{I}-\nabla_{0} \mathbf{u}^{T}\right)\right) \cdot \mathbf{n}_{0}\right]_{\Sigma_{0}}-\frac{1}{\left|\nabla_{0} S_{0}\right|} \nabla_{0} \cdot\left\{\left(\left|\nabla_{0} S_{0}\right|\left(\mathbf{A}_{0} \cdot \mathbf{n}_{0}\right) \otimes\left[\mathbf{u}_{\perp}\right]\right)_{\Sigma_{0}}\right\}=\mathbf{0}
$$

and:

$$
\left[\left(\mathbf{b}^{L}+\mathbf{b}_{0} \cdot\left(\left(\nabla_{0} \cdot \mathbf{u}\right) \mathbf{I}-\nabla_{0} \mathbf{u}^{T}\right)\right) \cdot \mathbf{n}_{0}\right]_{\Sigma_{0}}-\frac{1}{\left|\nabla_{0} S_{0}\right|} \nabla_{0} \cdot\left\{\left(\left|\nabla_{0} S_{0}\right|\left(\mathbf{b}_{0} \cdot \mathbf{n}_{0}\right)\left[\mathbf{u}_{\perp}\right]\right)_{\Sigma_{0}}\right\}=0
$$

with $\mathbf{u}_{\perp}=\mathbf{u}-\left(\mathbf{u} \cdot \mathbf{n}_{0}\right) \mathbf{n}_{0}$ defining the tangential displacement. Note that the last terms vanish in the no-slip case. These jump conditions generalize to curved interfaces the conditions of Poirée $[14,15]$, which were formally derived for plane interfaces only.

At this stage, it should be emphasized that the exactness of interface conditions (15), (17) and (18) has not yet been rigorously demonstrated. Their validity will be assessed in Sec. 4 .

\section{Validation}

One must be careful because the procedure of deriving interface conditions based on generalized function theory does not formally prove that the soobtained jump conditions are exact. A typical counter-example may be given by the Eulerian perturbation of mass conservation:

$$
\frac{\partial \rho^{E}}{\partial t}+\nabla_{0} \cdot\left(\rho^{E} \mathbf{v}_{0}+\rho_{0} \mathbf{v}^{E}\right)=0
$$

which is still written under a conservative form through the identification $\rho=\rho_{0}, \mathbf{v}=\mathbf{v}_{0}, b=\rho^{E} / \rho_{0}$ and $\mathbf{b}=\rho_{0} \mathbf{v}^{E}$ within Eq. (A.8). However, one can verify that applying into Eq. (19) a decomposition of the form (A.2) for $\rho_{0}, \rho^{E}, \mathbf{v}_{0}$ and $\mathbf{v}^{E}$ would give the jump condition $\left[\rho_{0} \mathbf{v}^{E} \cdot \mathbf{n}_{0}\right]_{\Sigma_{0}}=0$, which is obviously incorrect - a detailed study can be found in Poirée $[14,35]$.

As a central result of this paper, this section aims at verifying the validity of the previously obtained interface conditions for perturbations. A possible way of proceeding, which we follow, is to compare conditions (15), (17) and (18) to the ones obtained from an alternative approach based on an exact linearization of the standard Eulerian jump conditions. In order to ensure a straightforward comparison, all expressions are written in a curvilinear coordinate system attached to the interface. 


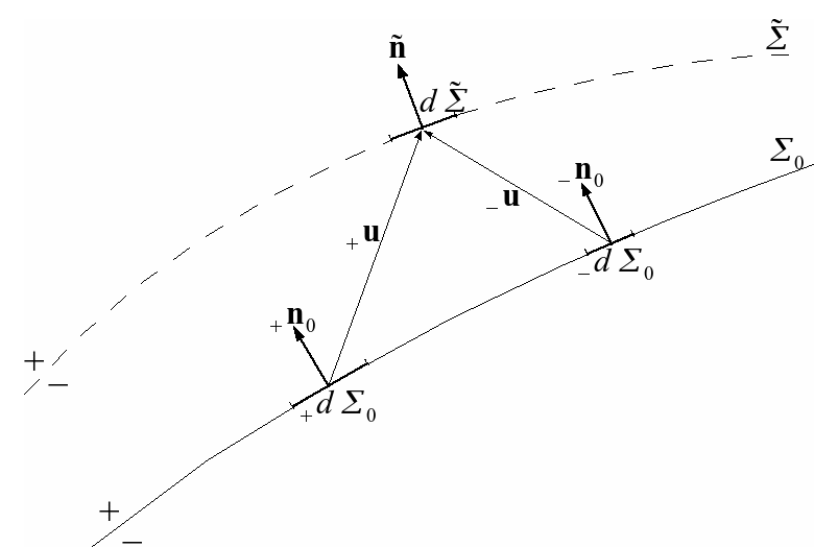

Fig. 3. Transformation of material surface elements in the presence of slip. $\Sigma_{0}$ and $\tilde{\Sigma}$ represent the interface in its intermediate (thin line) and current (dashed) configurations. In the no-slip case, $+\mathbf{u}=-\mathbf{u}$ and the intermediate surface elements would coincide.

\subsection{Alternative derivation (exact linearization)}

Jump conditions for perturbations are now derived based on an exact approach starting from the standard Eulerian conditions (13), rewritten as:

$$
{ }_{-} \tilde{\mathbf{A}} \cdot \tilde{\mathbf{n}} d \tilde{\Sigma}={ }_{+} \tilde{\mathbf{A}} \cdot \tilde{\mathbf{n}} d \tilde{\Sigma},{ }_{-} \tilde{\mathbf{b}} \cdot \tilde{\mathbf{n}} d \tilde{\Sigma}={ }_{+} \tilde{\mathbf{b}} \cdot \tilde{\mathbf{n}} d \tilde{\Sigma}
$$

The method consists in transforming the above conditions to the intermediate configuration thanks to the following formula, well-known in continuum mechanics and sometimes referred to as Nanson's formula: $\tilde{\mathbf{n}} d \tilde{\Sigma}=\operatorname{det}\left(\tilde{\mathbf{X}}_{0}\right) \tilde{\mathbf{X}}_{0}^{-T} \mathbf{n}_{0} d \Sigma_{0}$ $\left(\tilde{\mathbf{X}}_{0}\right.$ already defined in Sec. 2.5). In order to write conditions at the same geometrical point in the presence of slip, we must transform surface element on either side to the same intermediate element, that of medium + for instance. For clarity, Fig. 3 sketches the transformation of material surface elements in the presence of slip from the current to the intermediate configurations. The linearized Nanson's formula then yields up to the first order:

$$
\tilde{\mathbf{n}} d \tilde{\Sigma}=\mathbf{n}_{0} d \Sigma_{0}+\epsilon\left(\left(\nabla_{0} \cdot+\mathbf{u}\right) \mathbf{I}-\left(\nabla_{0+} \mathbf{u}\right)^{T}\right) \cdot \mathbf{n}_{0} d \Sigma_{0}
$$

where $\mathbf{n}_{0} d \Sigma_{0}$ should be understood as ${ }_{+} \mathbf{n}_{0+} d \Sigma_{0}$.

With this transformation, the perturbations of ${ }_{ \pm} \tilde{\mathbf{A}}$ denoted ${ }_{ \pm} \mathbf{A}^{P}$ are explicitly defined by ${ }_{-} \tilde{\mathbf{A}}(\tilde{\mathbf{x}})={ }_{-} \mathbf{A}_{0}\left({ }_{+} \mathbf{x}_{0}\right)+\epsilon_{-} \mathbf{A}^{P}$ and ${ }_{+} \tilde{\mathbf{A}}(\tilde{\mathbf{x}})={ }_{+} \mathbf{A}_{0}\left({ }_{+} \mathbf{x}_{0}\right)+\epsilon_{+} \mathbf{A}^{P}$. From definition (5), it is easy to remark that ${ }_{+} \mathbf{A}^{P}={ }_{+} \mathbf{A}^{L}$ and ${ }_{-} \mathbf{A}^{P}={ }_{-} \mathbf{A}^{L}+$ $\left([\mathbf{u}] \cdot \nabla_{0}\right)_{-} \mathbf{A}_{0}$ (with similar relationships for ${ }_{ \pm} \mathbf{b}^{P}$ ). Making use of these results and of the intermediate state condition $\left({ }_{+} \mathbf{A}_{0}-{ }_{-} \mathbf{A}_{0}\right) \cdot \mathbf{n}_{0} d \Sigma_{0}=\mathbf{0}$, the exact linearization of Eqs. (20) gives the following jump conditions for perturbations:

$$
\left\{\left[\mathbf{A}^{L}\right]+\left[\mathbf{A}_{0}\right] \cdot\left(\left(\nabla_{0} \cdot+\mathbf{u}\right) \mathbf{I}-\left(\nabla_{0+} \mathbf{u}\right)^{T}\right)-\left([\mathbf{u}] \cdot \nabla_{0}\right)_{-} \mathbf{A}_{0}\right\} \cdot \mathbf{n}_{0}=\mathbf{0} \text { on } \Sigma_{0}
$$


with a similar expression for a scalar condition (just replace $\mathbf{A}$ with $\mathbf{b}$ ).

\subsection{Sufficiency of the kinematic condition (15)}

The sufficiency of the normal Lagrangian displacement continuity (15) is now proved. One needs to consider a curvilinear coordinate system, denoted $\left(\xi^{1}, \xi^{2}, \xi^{3}\right)$, such that the interface $\Sigma_{0}$ defined by the implicit equation $S_{0}\left(\mathbf{x}_{0}(t), t\right)=$ 0 is given by $\xi^{3}=f(t)$ in terms of these new coordinates. The corresponding covariant basis $\left(\mathbf{g}_{1}, \mathbf{g}_{2}, \mathbf{g}_{3}\right)$ is given by $\mathbf{g}_{i}=\partial \mathbf{x}_{0} / \partial \xi^{i}(i=1,2,3)$, the covariant metric tensor is $g_{i j}=\mathbf{g}_{i} \cdot \mathbf{g}_{j}$. Then, $\mathbf{g}_{\alpha}(\alpha=1,2)$ are tangent vectors (assumed linearly independent) and $\mathbf{g}_{3}$ is a normal vector to the interface. The contravariant $\left(\mathbf{g}^{1}, \mathbf{g}^{2}, \mathbf{g}^{3}\right)$ basis is defined by $\mathbf{g}_{i} \cdot \mathbf{g}^{j}=\delta_{i}^{j}\left(\delta_{i}^{j}\right.$ being the Kronecker symbol), $g^{i j}=\mathbf{g}^{i} \cdot \mathbf{g}^{j}$ being its metric tensor. The following notation conventions are used: right subscripts (resp. superscripts) will denote covariant (resp. contravariant) components, latin (resp. Greek) indices will be used for 3D (resp. surface) components. We will use Einstein summation convention and commas for partial derivatives with respect to curvilinear coordinates. The following properties hold: $g_{\alpha 3}=g^{\alpha 3}=0$ and $\mathbf{n}_{0}=\mathbf{g}_{3} /\left|\mathbf{g}_{3}\right|=\mathbf{g}^{3} /\left|\mathbf{g}^{3}\right|$.

The main properties and expressions of operators used in this paper are recalled in Appendix B. For instance, the reader may refer to [36-38] for an introduction to the use of general curvilinear coordinate systems.

One starts from Eq. (22) written for the velocity. This yields an exact but cumbersome kinematic condition. As shown by the following Lemma, it can be greatly simplified when expressed in the curvilinear coordinate system thanks to the use of jump compatibility conditions (B.4) and (B.5).

Lemma 4.1 The linearized interface condition associated with the continuity of normal velocity $[\tilde{\mathbf{v}} \cdot \tilde{\mathbf{n}}]_{\tilde{\Sigma}}=0$ can be expressed in terms of the Lagrangian perturbation of displacement as:

$$
\left(\frac{d_{0}}{d t}-\mathbf{n}_{0} \cdot \nabla_{0}\left(\left(\mathbf{v}_{0} \cdot \mathbf{n}_{0}\right) \cdot\right)\right)\left(\left[\mathbf{u} \cdot \mathbf{n}_{0}\right]_{\Sigma_{0}}\right)=0
$$

Proof. Recall that $\mathbf{v}^{L}=d_{0} \mathbf{u} / d t$, Eqs. (22) written for the velocity $(\mathbf{b}=\mathbf{v})$ yields a kinematic condition written in terms of the displacement only:

$$
\left\{\left[d_{0} \mathbf{u} / d t\right]+\left[\mathbf{v}_{0}\right] \cdot\left(\left(\nabla_{0} \cdot+\mathbf{u}\right) \mathbf{I}-\left(\nabla_{0}+\mathbf{u}\right)^{T}\right)-\left([\mathbf{u}] \cdot \nabla_{0}\right)_{-} \mathbf{v}_{0}\right\} \cdot \mathbf{n}_{0}=0 \text { on } \Sigma_{0}
$$

Using Appendix B and the continuity of intermediate normal velocity $\left[v_{0}^{3}\right]_{\Sigma_{0}}=$ 0 , some developments and simplifications yield the following equality on $\Sigma_{0}$ : 


$$
\begin{array}{r}
\left\{\left[\left(\mathbf{v}_{0} \cdot \nabla_{0}\right) \mathbf{u}\right]+\left[\mathbf{v}_{0}\right] \cdot\left(\left(\nabla_{0} \cdot+\mathbf{u}\right) \mathbf{I}-\left(\nabla_{0+} \mathbf{u}\right)^{T}\right)-\left([\mathbf{u}] \cdot \nabla_{0}\right)_{-} \mathbf{v}_{0}\right\} \cdot \mathbf{n}_{0} \\
=\left({ }_{-} v_{0}^{i}\left[u_{, i}^{3}\right]-{ }_{-} v_{0, i}^{3}\left[u^{i}\right]\right) /\left|\mathbf{g}^{3}\right|
\end{array}
$$

The first term of the material derivative in Eq. (24) requires some specific developments for a non-stationary interface $\Sigma_{0}$ and is decomposed as follows:

$\left[\frac{\partial \mathbf{u}}{\partial t}\right] \cdot \mathbf{n}_{0}=\left[\frac{\partial\left(\mathbf{u} \cdot \mathbf{n}_{0}\right)}{\partial t}-\mathbf{u} \cdot \frac{\partial \mathbf{n}_{0}}{\partial t}\right]=\left[\frac{\partial}{\partial t}\left(\frac{u^{3}}{\left|\mathbf{g}^{3}\right|}\right)\right]-\left[u^{i} \mathbf{g}_{i} \cdot \frac{\partial}{\partial t}\left(\frac{\mathbf{g}^{3}}{\left|\mathbf{g}^{3}\right|}\right)\right]$ on $\Sigma_{0}$

We have $\mathbf{n}_{0} \cdot \partial \mathbf{n}_{0} / \partial t=0$ (from $\mathbf{n}_{0} \cdot \mathbf{n}_{0}=1$ ) and hence $\mathbf{g}_{3} \cdot \partial\left(\mathbf{g}^{3} /\left|\mathbf{g}^{3}\right|\right) / \partial t=0$. Recall the jump kinematic compatibility condition given by Eq. (B.5), Eq. (26) now becomes:

$$
\left[\frac{\partial \mathbf{u}}{\partial t}\right]_{\Sigma_{0}} \cdot \mathbf{n}_{0}=\frac{\partial}{\partial t}\left(\frac{\left[u^{3}\right]_{\Sigma_{0}}}{\left|\mathbf{g}^{3}\right|}\right)-w_{0}^{3}\left[\left(\frac{u^{3}}{\left|\mathbf{g}^{3}\right|}\right)_{, 3}\right]_{\Sigma_{0}}-\left[u^{\alpha} \mathbf{g}_{\alpha} \cdot \frac{\partial}{\partial t}\left(\frac{\mathbf{g}^{3}}{\left|\mathbf{g}^{3}\right|}\right)\right]_{\Sigma_{0}}
$$

where $w_{0}^{3}=w_{n_{0}}\left|\nabla_{0} S_{0}\right|$ ( $w_{n_{0}}$ being the intermediate surface normal velocity). From the relation $\mathbf{g}^{3}=\nabla_{0} S_{0}$, we have $\partial \mathbf{g}^{3} / \partial t=-\nabla_{0} w_{0}^{3}=-w_{0, i}^{3} \mathbf{g}^{i}$. Summing Eqs. (25) and (27) and using (B.6), the condition (24) might finally be rewritten as:

$$
\left(\frac{\partial \cdot}{\partial t}+{ }_{-} v_{0}^{\alpha}(\cdot)_{, \alpha}-{ }_{-} v_{0,3}^{3} \cdot-\Gamma_{33}^{3} w_{0}^{3} \cdot\right)\left(\frac{\left[u^{3}\right]_{\Sigma_{0}}}{\left|\mathbf{g}^{3}\right|}\right)=\frac{w_{0}^{3}-{ }_{-} v_{0}^{3}}{\left|\mathbf{g}^{3}\right|}\left[u_{, 3}^{3}\right]_{\Sigma_{0}}+\frac{\left(-v_{0}^{3}-w_{0}^{3}\right)_{, \alpha}}{\left|\mathbf{g}^{3}\right|}\left[u^{\alpha}\right]_{\Sigma_{0}}
$$

The right-hand side is zero thanks to the continuity of normal velocity in the intermediate state $\left(w_{0}^{3}={ }_{-} v_{0}^{3}\right)$ and the geometric compatibility condition (B.4). The left-hand side can be rewritten in the condensed form given by Eq. (23). Note that the above equality also holds for ${ }_{+} v_{0}^{i}$ instead of $v_{0}^{i}$. This ends the proof of Lemma 4.1.

Theorem 4.2 Assume an interface across which the normal velocity remains continuous: $\left[\mathbf{v}_{0} \cdot \mathbf{n}_{0}\right]_{\Sigma_{0}}=[\tilde{\mathbf{v}} \cdot \tilde{\mathbf{n}}]_{\tilde{\Sigma}}=0$. A sufficient condition for small perturbations is given by the continuity of the normal Lagrangian perturbation of displacement: $\left[\mathbf{u} \cdot \mathbf{n}_{0}\right]_{\Sigma_{0}}=0$.

The proof is straightforward from the exact linearized condition (23) of Lemma 4.1, which is necessarily satisfied when the continuity $\left[\mathbf{u} \cdot \mathbf{n}_{0}\right]_{\Sigma_{0}}=0$ is assumed.

Note that the continuity of the normal Lagrangian perturbation of displacement is generally also a necessary condition, except when the left-hand side differential operator of Eq. (23) vanishes. This situation may occur for special cases of wave-flow synchronim, as already stated by Godin $[16,17]$ when considering stationary intermediate interfaces (for a stationary interface, $\partial S_{0} / \partial t=$ $\left.w_{0}^{3}=\left.v_{0}^{3}\right|_{\Sigma_{0}}=0\right)$. In this paper, note that no stationarity assumption has been required for the intermediate interface in the derivation of Eq. (23). 
One first needs to write the exact linearized interface conditions (22) in the curvilinear coordinate system. The curvilinear expressions are given by the following Lemma:

Lemma 4.3 Based on the condition $\left[\mathbf{u} \cdot \mathbf{n}_{0}\right]_{\Sigma_{0}}=0$, the linearized interface condition obtained from the Eulerian condition $[\tilde{\mathbf{A}} \cdot \tilde{\mathbf{n}}]_{\tilde{\Sigma}}=\mathbf{0}$ (where $\tilde{\mathbf{A}}$ is a second order tensor) can be expressed as:

$$
\left[A^{L i 3}-A_{0, \alpha}^{i 3} u^{\alpha}-A_{0}^{i \alpha}\left(u_{, \alpha}^{3}+\Gamma_{\alpha k}^{3} u^{k}\right)-A_{0}^{j 3} \Gamma_{j \alpha}^{i} u^{\alpha}-A_{0}^{i 3} \Gamma_{3 \alpha}^{3} u^{\alpha}\right]_{\Sigma_{0}}=0
$$

(for $i=1,2,3$ ) in a curvilinear coordinate system attached to the interface $\Sigma_{0}$, represented by the implicit equation $\xi^{3}=f(t)$. Similarly, the linearized interface condition obtained from $[\tilde{\mathbf{b}} \cdot \tilde{\mathbf{n}}]_{\tilde{\Sigma}}=0$ ( $\tilde{\mathbf{b}}$ is a vector) is:

$$
\left[b^{L 3}-b_{0, \alpha}^{3} u^{\alpha}-b_{0}^{\alpha}\left(u_{, \alpha}^{3}+\Gamma_{\alpha k}^{3} u^{k}\right)-b_{0}^{3} \Gamma_{3 \alpha}^{3} u^{\alpha}\right]_{\Sigma_{0}}=0
$$

Proof. Thanks to Appendix B, the reader can check (details not shown for conciseness) that writing the condition (22) in the curvilinear coordinate system exactly yields Eqs. (29) and (30) provided that $\left[u^{3}\right]_{\Sigma_{0}}=0$.

Note that such expressions might be particularly suitable for practical applications involving curved interfaces.

Theorem 4.4 Based on the condition $\left[\mathbf{u} \cdot \mathbf{n}_{0}\right]_{\Sigma_{0}}=0$, the interface conditions (17) and (18) derived from generalized function theory are equivalent to the exact linearized conditions (22).

Proof. In the curvilinear coordinate system, conditions (13) in the intermediate state and the condition (15) are simply given by:

$$
\left[A_{0}^{i 3}\right]_{\Sigma_{0}}=0,\left[b_{0}^{3}\right]_{\Sigma_{0}}=0,\left[u^{3}\right]_{\Sigma_{0}}=0
$$

Conditions (17) and (18) require further developments. Using expressions of Appendix B with $\mathbf{n}_{0}=\mathbf{g}^{3} /\left|\mathbf{g}^{3}\right|$, we can write after simplifications:

$$
\begin{array}{r}
\left(\mathbf{A}^{L}+\mathbf{A}_{0} \cdot\left(\left(\nabla_{0} \cdot \mathbf{u}\right) \mathbf{I}-\nabla_{0} \mathbf{u}^{T}\right)\right) \cdot \mathbf{n}_{0}= \\
\left(A^{L i 3}+A_{0}^{i 3}\left(u_{, \alpha}^{\alpha}+\Gamma_{\alpha k}^{\alpha} u^{k}\right)-A_{0}^{i \alpha}\left(u_{, \alpha}^{3}+\Gamma_{\alpha k}^{3} u^{k}\right)\right) \mathbf{g}_{i} /\left|\mathbf{g}^{3}\right|
\end{array}
$$

Thanks to the relation (B.3) and $\left[u^{3}\right]_{\Sigma_{0}}=0$, we have:

$$
\left(\left|\nabla_{0} S_{0}\right|\left(\mathbf{A}_{0} \cdot \mathbf{n}_{0}\right) \otimes\left[\mathbf{u}_{\perp}\right]\right)_{\Sigma_{0}}=\left(A_{0}^{i 3}\left[u^{\alpha}\right] \mathbf{g}_{i} \otimes \mathbf{g}_{\alpha}\right)_{\Sigma_{0}}
$$


Table 2

Mass, momentum, energy and entropy interface conditions for perturbations.

\begin{tabular}{|c|c|}
\hline Mass & {$\left[\mathbf{u} \cdot \mathbf{n}_{0}\right]_{\Sigma_{0}}=0$} \\
\hline Momentum & $\begin{array}{l}{\left[\left(\boldsymbol{\sigma}^{L}+\boldsymbol{\sigma}_{0} \cdot\left(\left(\nabla_{0} \cdot \mathbf{u}\right) \mathbf{I}-\nabla_{0} \mathbf{u}^{T}\right)\right) \cdot \mathbf{n}_{0}\right]_{\Sigma_{0}}} \\
\quad-\frac{1}{\left|\nabla_{0} S_{0}\right|} \nabla_{0} \cdot\left\{\left(\left|\nabla_{0} S_{0}\right|\left(\boldsymbol{\sigma}_{0} \cdot \mathbf{n}_{0}\right) \otimes\left[\mathbf{u}_{\perp}\right]\right)_{\Sigma_{0}}\right\}=\mathbf{0}\end{array}$ \\
\hline Energy $^{1}$ & $\begin{array}{l}{\left[\left(\mathbf{q}^{L}+\mathbf{q}_{0} \cdot\left(\left(\nabla_{0} \cdot \mathbf{u}\right) \mathbf{I}-\nabla_{0} \mathbf{u}^{T}\right)\right) \cdot \mathbf{n}_{0}\right]_{\Sigma_{0}}} \\
\quad-\frac{1}{\left|\nabla_{0} S_{0}\right|} \nabla_{0} \cdot\left\{\left(\left|\nabla_{0} S_{0}\right|\left(\mathbf{q}_{0} \cdot \mathbf{n}_{0}\right)\left[\mathbf{u}_{\perp}\right]\right)_{\Sigma_{0}}\right\}=0\end{array}$ \\
\hline Entropy $^{2}$ & {$\left[T^{L}+\mathbf{u}_{\perp} \cdot \nabla_{0} T_{0}\right]_{\Sigma_{0}}=0$} \\
\hline
\end{tabular}

and the following expression holds:

$\nabla_{0} \cdot\left\{\left(\left|\nabla_{0} S_{0}\right|\left(\mathbf{A}_{0} \cdot \mathbf{n}_{0}\right) \otimes\left[\mathbf{u}_{\perp}\right]\right)_{\Sigma_{0}}\right\}=\left(\left(A_{0}^{i 3}\left[u^{\alpha}\right]\right)_{, \alpha}+\Gamma_{j \alpha}^{i} A_{0}^{j 3}\left[u^{\alpha}\right]+\Gamma_{\alpha j}^{j} A_{0}^{i 3}\left[u^{\alpha}\right]\right)_{\Sigma_{0}} \mathbf{g}_{i}$

Then from Eqs. (32) and (34) and thanks to Eqs. (31) as well as the geometric compatibility condition (B.4) and property (B.6), condition (17) may finally be rewritten as Eq. (29). Following the same method, the curvilinear expression for the scalar interface condition (18) is shown to be the same as Eq. (30). This ends the proof of Theorem 4.4.

\section{Discussions}

\subsection{Application to mass, momentum, energy and entropy equations}

Table 2 gives the interface conditions derived in Sec.3.2 for mass (kinematic condition), momentum (dynamic condition), energy and entropy, yielding equations for the perturbations of displacement, stress, heat flux and temperature.

The jump condition for stress is obtained by applying the identification (11) into Eq. (17). As far as energy and entropy are concerned, the expression of jump conditions are more cumbersome. To the authors' knowledge, general expressions of jump conditions for perturbations of heat flux and temperature have not been proposed in the literature. Some general conditions can be obtained from the identification (11) into Eq. (18). However for conciseness, Table 2 only gives simplified expressions of the energy and entropy conditions, as detailed below.

When the interface is bonded $\left(\left[\mathbf{v}_{0}\right]_{\Sigma_{0}}=[\mathbf{u}]_{\Sigma_{0}}=\left[\mathbf{v}^{L}\right]_{\Sigma_{0}}=\mathbf{0}\right)$ or involves

\footnotetext{
1 assumption: the interface is bonded or involves an inviscid fluid.

2 assumptions: same as footnote ${ }^{1}$ and the temperature remains continuous across the interface.
} 
an inviscid fluid $\left(\boldsymbol{\sigma}_{0} \cdot \mathbf{n}_{0}=-p_{0} \mathbf{n}_{0}, \boldsymbol{\sigma}^{L} \cdot \mathbf{n}_{0}=-p^{L} \mathbf{n}_{0}\right)$, the energy jump condition can be simplified through the use of the kinematic and dynamic jump conditions. It can be checked that one arrives at the energy condition given in Table 2. Recall that $\left[\mathbf{q}_{0} \cdot \mathbf{n}_{0}\right]_{\Sigma_{0}}=0$. Let us further assume an interface across which the temperature is continuous $\left([\tilde{T}]_{\tilde{\Sigma}}=\left[T_{0}\right]_{\Sigma_{0}}=0\right)$. Then using the energy condition, the entropy jump condition degenerates into the expression given in Table 2.

Note that for a bonded interface, the respective last terms of momentum, energy and entropy jump conditions vanish. In particular, this yields the continuity of temperature Lagrangian perturbation $\left[T^{L}\right]_{\Sigma_{0}}=0\left(\neq\left[T^{E}\right]_{\Sigma_{0}}\right.$ in general $)$.

\subsection{Myers approach for Eulerian perturbations}

Let us consider the kinematic continuity condition $\left[\mathbf{u} \cdot \mathbf{n}_{0}\right]_{\Sigma_{0}}=0$. It involves the Lagrangian perturbation of the displacement, not suited with the fully Eulerian approach usually used in fluid acoustics. When no initial flow is present, this condition is obviously equivalent to $\left[\mathbf{v}^{E} \cdot \mathbf{n}_{0}\right]_{\Sigma_{0}}=0$. However this is not the case anymore with a flowing fluid, for which the Eulerian kinematic boundary condition was found by Myers [12] under the assumption of a stationary intermediate interface. Its approach consisted in linearizing the condition $[\tilde{\mathbf{v}} \cdot \tilde{\mathbf{n}}]_{\tilde{\Sigma}}=0$ by means of Eulerian perturbations (instead of Lagrangian perturbations as done in Sec. 4.1).

For clarity, the kinematic condition of Myers is with our notations:

$$
\mathbf{v}^{E} \cdot \mathbf{n}_{0}=\partial \zeta / \partial t+\mathbf{v}_{0} \cdot \nabla_{0} \zeta-\zeta \mathbf{n}_{0} \cdot\left(\mathbf{n}_{0} \cdot \nabla_{0}\right) \mathbf{v}_{0} \text { on } \Sigma_{0}
$$

where $\zeta$ is defined as the normal displacement of the interface. As mentioned in the introduction, the equivalence between the normal Lagrangian displacement continuity and Myers condition has already been proved by Godin [16,17], who has shown that $\zeta=\left.\mathbf{u} \cdot \mathbf{n}_{0}\right|_{\Sigma_{0}}$ implies Myers condition. In this paper, Lemma 4.1 and Theorem 4.2 can be viewed as an extension of Godin's proof for non-stationary interfaces. What should be remarked is that the normal Lagrangian displacement indeed naturally appear in Myers condition also (this condition is thus not fully Eulerian).

In the no-slip case, $[\tilde{\mathbf{v}}]_{\tilde{\Sigma}}=\mathbf{0}$ and the following continuity conditions obviously hold: $\left[\mathbf{v}^{L}\right]_{\Sigma_{0}}=[\mathbf{u}]_{\Sigma_{0}}=\mathbf{0}$. However, from the relation (6), $\left[\mathbf{v}^{E}\right]_{\Sigma_{0}}=-[(\mathbf{u}$. $\left.\left.\nabla_{0}\right) \mathbf{v}_{0}\right]_{\Sigma_{0}}=-u^{3}\left[v_{0,3}^{i}\right]_{\Sigma_{0}} \mathbf{g}_{i}$ : this jump is generally non-zero. In the case of a stationary bonded interface, the Myers-type condition would be written as $\mathbf{v}^{E}=\partial \boldsymbol{\zeta} / \partial t-\left(\boldsymbol{\zeta} \cdot \mathbf{n}_{0}\right)\left(\mathbf{n}_{0} \cdot \nabla_{0}\right) \mathbf{v}_{0}$ on $\Sigma_{0}$ (where $\boldsymbol{\zeta}=\left.\mathbf{u}\right|_{\Sigma_{0}}$ now corresponds to the interface displacement vector). 
Table 3

Myers-type interface conditions for Eulerian perturbations ( $\zeta$ is defined as the interface normal displacement).

\begin{tabular}{ll}
\hline Kinematic condition & {$\left[\mathbf{v}^{E} \cdot \mathbf{n}_{0}-\mathbf{v}_{0} \cdot \nabla_{0} \zeta+\zeta\left(\nabla_{0} \mathbf{v}_{0} \cdot \mathbf{n}_{0}\right) \cdot \mathbf{n}_{0}\right]_{\Sigma_{0}}=0$} \\
\hline Dynamic condition & {$\left[\boldsymbol{\sigma}^{E} \cdot \mathbf{n}_{0}-\boldsymbol{\sigma}_{0} \cdot \nabla_{0} \zeta+\zeta\left(\nabla_{0} \boldsymbol{\sigma}_{0} \cdot \mathbf{n}_{0}\right) \cdot \mathbf{n}_{0}\right]_{\Sigma_{0}}=\mathbf{0}$} \\
\hline
\end{tabular}

Table 4

Dynamic interface conditions for perturbations in the literature.

\begin{tabular}{ll}
\hline $\begin{array}{l}\text { Authors } \\
\text { (interaction type) }\end{array}$ & $\begin{array}{l}\text { Dynamic jump condition } \\
\text { for perturbations }\end{array}$ \\
\hline \hline Norris et al. [20] & {$\left[\mathbf{P} \cdot \mathbf{n}_{0}-\left(\nabla_{0} \cdot \mathbf{u}-\mathbf{n}_{0} \cdot\left(\nabla_{0} \mathbf{u}\right) \cdot \mathbf{n}_{0}\right) \boldsymbol{\sigma}_{0} \cdot \mathbf{n}_{0}\right.$} \\
(general) & $\left.-\left(\mathbf{u}_{\perp} \cdot \nabla_{0}\right)\left(\boldsymbol{\sigma}_{0} \cdot \mathbf{n}_{0}\right)\right]_{\Sigma_{0}}=\mathbf{0}$ \\
\hline Schotté and Ohayon [19] & $+\boldsymbol{\sigma}=-\left(-p^{L}+[\mathbf{u}] \cdot \nabla_{0-} p_{0}\right) \mathbf{n}_{0}$ \\
(fluid-structure) $^{3}$ & $-{ }_{-} p_{0}\left(\left(\nabla_{0} \cdot+\mathbf{u}\right) \mathbf{I}-\left(\nabla_{0+} \mathbf{u}\right)^{T}\right) \cdot \mathbf{n}_{0} \quad$ on $\Sigma_{0}$ \\
\hline Godin [17] (fluid-fluid) $^{3}$ & {$\left[p^{E}+\left(\mathbf{u} \cdot \mathbf{n}_{0}\right) \mathbf{n}_{0} \cdot \nabla_{0} p_{0}\right]_{\Sigma_{0}}=0$} \\
\hline
\end{tabular}

Following the same linearization method as Myers, a kinematic condition can be obtained in a jump form for non-stationary intermediate interfaces. This condition is given in Table 3. This approach could also be used to derive Eulerian perturbations of jump conditions for stress, energy and entropy (indeed, simply obtained by replacing the letter $\mathbf{v}$ with fields $\mathbf{A}$ or $\mathbf{b}$ in the kinematic condition of Table 3) - as an example, Table 3 gives the dynamic condition. Following the same procedure as in Sec. 4.2, one can check that $\zeta=\left.{ }_{ \pm} \mathbf{u} \cdot \mathbf{n}_{0}\right|_{\Sigma_{0}}$ is a sufficient condition for the linearized kinematic condition of Table 3 to be satisfied (which is equivalent to Theorem 4.2). Furthermore, provided that $\zeta=\left.\mathbf{u} \cdot \mathbf{n}_{0}\right|_{\Sigma_{0}}$ and using the relation $\boldsymbol{\sigma}^{E}=\boldsymbol{\sigma}^{L}-\left(\mathbf{u} \cdot \nabla_{0}\right) \boldsymbol{\sigma}_{0}$, one can also check that writing the dynamic condition of Table 3 in a curvilinear coordinate system exactly yields the form given by Eq. (29) in Lemma 4.3.

However, conditions obtained with the mixed Eulerian-Lagrangian description remain rather simple (kinematic conditions particularly) while Eulerian Myers-type conditions, indeed not fully Eulerian, are more difficult to write and implement in practice. This makes the mixed formulation particularly attractive for interaction problem involving acoustics in the presence of initial flow and/or prestress. For finite element applications of kinematic conditions in flow acoustics, see for instance Refs. [32,39-45].

\subsection{Literature results for the dynamic condition}

Table 4 gives some standard literature results for the dynamic condition. 
Let us consider the dynamic condition in Table 2. From the expressions of Appendix B and the property (B.6), it can be verified that the following identity holds:

$\frac{1}{\left|\nabla_{0} S_{0}\right|} \nabla_{0} \cdot\left\{\left(\left|\nabla_{0} S_{0}\right|\left[\mathbf{u}_{\perp}\right]\right)_{\Sigma_{0}}\right\}=\left(\left[u_{, \alpha}^{\alpha}\right]+\Gamma_{\beta \alpha}^{\beta}\left[u^{\alpha}\right]\right)_{\Sigma_{0}}=\left[\nabla_{0} \cdot \mathbf{u}-\mathbf{n}_{0} \cdot\left(\nabla_{0} \mathbf{u}\right) \cdot \mathbf{n}_{0}\right]_{\Sigma_{0}}$

This allows us to rewrite the dynamic condition of Table 2 as the jump condition of Norris [20] generalized to non-uniform prestress along the interface, given in Table 4 with the new stress notation: $\mathbf{P}=\boldsymbol{\sigma}^{L}+\boldsymbol{\sigma}_{0} \cdot\left(\left(\nabla_{0} \cdot \mathbf{u}\right) \mathbf{I}-\nabla_{0} \mathbf{u}^{T}\right)$. Note that this condition remains unchanged in the presence of initial flow.

The stress jump conditions derived in this paper also coincides with the condition obtained by Schotté and Ohayon [19] between an inviscid fluid that is initially at rest and and an elastic prestressed structure (see Table 4). These authors indeed used a linearization process similar to Sec. 4.1. Their condition can be recovered from Eq. (22) (with $\mathbf{A}^{L}=\boldsymbol{\sigma}^{L}$ and $\mathbf{A}_{0}=\boldsymbol{\sigma}_{0}$ ) expressed in terms of the increment ${ }_{+} \boldsymbol{\sigma}$ as defined by Eq. (12) rather than ${ }_{+} \boldsymbol{\sigma}^{L}(-$ and + signs denoting the fluid and solid media respectively).

Finally, for an inviscid fluid-fluid problem, we have $\boldsymbol{\sigma}_{0}=-p_{0} \mathbf{I}$ and $\boldsymbol{\sigma}^{L}=-p^{L} \mathbf{I}$ so that: $\sigma_{0}^{i j}=-p_{0} g^{i j}$ and $\sigma^{L i j}=-p^{L} g^{i j}$. Replacing this expression into the curvilinear condition (29) yields:

$p_{0}\left(g^{\gamma \beta} \Gamma_{\beta \alpha}^{3}+g^{33} \Gamma_{3 \alpha}^{\gamma}\right)\left[u^{\alpha}\right]_{\Sigma_{0}}=0, \quad\left[p^{L}-u^{\alpha} p_{0, \alpha}\right]_{\Sigma_{0}}=p_{0}\left[u^{\alpha}\right]_{\Sigma_{0}}\left(g_{, \alpha}^{33}+2 g^{33} \Gamma_{3 \alpha}^{3}\right) / g^{33}$

The term inside parenthesis of the first equation vanishes thanks to Eq. (B.7) yielding zero on both sides. The right hand side of the second equation also vanishes thanks to Eq. (B.6), yielding the simple condition: $\left[p^{L}-u^{\alpha} p_{0, \alpha}\right]_{\Sigma_{0}}=0$ or in condensed form:

$$
\left[p^{L}-\mathbf{u}_{\perp} \cdot \nabla_{0} p_{0}\right]_{\Sigma_{0}}=0
$$

From relation (6), the above condition can also be written in terms of the Eulerian pressure, as the expression proposed by Godin [17] recalled in Table 4.

\section{Conclusion}

In this paper, the analysis of jump conditions for linear unsteady perturbations has been conducted at impermeable interfaces (slip or bonded, plane or not) between fluids and/or structures in the presence of initial flow and prestress. It has been shown that some general and valid conditions can be naturally

$\overline{3}$ assumption: inviscid fluid 
derived from the interpretation as generalized functions of the conservative equilibrium equations obtained with a mixed Eulerian-Lagrangian description.

The jump conditions for mass, momentum, energy and entropy have been discussed, yielding conditions for the Lagrangian perturbations of displacement, stress, heat flux and temperature. The obtained kinematic jump condition is quite simple and corresponds to the normal Lagrangian displacement continuity across the interface. The sufficiency of such a condition has been proved without requiring the assumption of initial stationarity of the interface. It has also been verified that the proposed stress jump conditions coincides with literature results.

The mixed Eulerian-Lagrangian description allows an interesting unification between fluid and solid formulations. For solids, this description yields the linearized updated Lagrangian formulation, well-known in non-linear mechanics, describing the linear dynamics of prestressed structures. For inviscid fluids, it gives the so-called Galbrun's equation, written in terms of the Lagrangian displacement only, describing sound propagation in arbitrary non-uniform Eulerian flows. For problems involving acoustics, such a formulation may yield simpler jump conditions than a fully Eulerian description, for which jump conditions explicitly involve the interface normal displacement.

\section{Acknowledgements}

The authors wish to thank Bernard Poirée for stimulating and helpful discussions.

\section{A Derivation of jump conditions from conservative equations}

For the sake of generality in this appendix, the notation convention using ref, 0 and tilde is dropped. A vector equilibrium equation has then the following general conservative form:

$$
\frac{\partial(\rho \mathbf{a})}{\partial t}+\nabla \cdot(\rho \mathbf{a} \otimes \mathbf{v}+\mathbf{A})=\rho \boldsymbol{\alpha}
$$

where $\mathbf{a}, \boldsymbol{\alpha}$ are vectors and $\mathbf{A}$ is a second order tensor. One postulates that Eq. (A.1) is valid in the sense of distributions and interprets all derivatives as generalized derivatives [22,23]. As stated by Farassat [22], it must be emphasized that the conservative form of a balance equation is particularly suitable with the use of generalized functions (with non-conservative forms, one may face with ambiguities of multiplication of generalized functions). 
Every physical quantity may be discontinuous at the smooth interface $\Sigma$, represented by the implicit equation $S(\mathbf{x}, t)=0$, so that we can write the following decompositions:

$$
\{\rho, \mathbf{v}, \mathbf{a}, \boldsymbol{\alpha}\}=\left\{{ }_{-} \rho,_{-} \mathbf{v},_{-} \mathbf{a},_{-} \boldsymbol{\alpha}\right\} H(-S)+\left\{{ }_{+} \rho,_{+} \mathbf{v},_{+} \mathbf{a},_{+} \boldsymbol{\alpha}\right\} H(+S)
$$

and:

$$
\mathbf{A}={ }_{-} \mathbf{A} H(-S)+{ }_{+} \mathbf{A} H(+S)+{ }_{\delta} \mathbf{A} \delta(S)
$$

with $H$ denoting the Heaviside function and $\delta$ the Dirac distribution. The Dirac term ${ }_{\delta} \mathbf{A}$ inside Eq. (A.3) (only defined on $S=0$ ) might represent some surface phenomena (for instance, superficial tension effect). Though such phenomena are neglected in this paper, the presence of this term will be useful for the derivation of jump conditions for perturbations in Sec. 3.2.

Time and spatial derivatives of $H( \pm S)$ are given by:

$$
\frac{\partial}{\partial t}(H( \pm S))=\mp w_{n}|\nabla S| \delta(S), \nabla(H( \pm S))= \pm \mathbf{n}|\nabla S| \delta(S)
$$

where $w_{n}$ is the normal velocity of the surface $\Sigma$. The time derivative has been obtained from the kinematic equation $\partial S / \partial t+\partial \mathbf{y} / \partial t \cdot \nabla S=0$ (y denoting any point on the surface $\Sigma$ ).

Substituting the expressions (A.2) and (A.3) into (A.1), using equalities (A.4) as well as the identities $H( \pm S) H( \pm S)=H( \pm S)$ and $H(+S) H(-S)=0$, we finally get the following equation:

$$
{ }_{-} \mathbf{E} H(-S)+{ }_{+} \mathbf{E} H(+S)+{ }_{\delta} \mathbf{E}|\nabla S| \delta(S)+{ }_{\delta^{\prime}} \mathbf{E}|\nabla S| \delta^{\prime}(S)=\mathbf{0}
$$

with the notations:

$$
{ }_{ \pm} \mathbf{E}=\frac{\partial\left({ }_{ \pm} \rho_{ \pm} \mathbf{a}\right)}{\partial t}+\nabla \cdot\left({ }_{ \pm} \rho_{ \pm} \mathbf{a} \otimes{ }_{ \pm} \mathbf{v}+{ }_{ \pm} \mathbf{A}\right)-{ }_{ \pm} \rho_{ \pm} \boldsymbol{\alpha}
$$

and:

$$
{ }_{\delta} \mathbf{E}=\left[\rho \mathbf{a}\left(\mathbf{v} \cdot \mathbf{n}-w_{n}\right)+\mathbf{A} \cdot \mathbf{n}\right]+\frac{1}{|\nabla S|} \nabla \cdot{ }_{\delta} \mathbf{A},{ }_{{ }^{\prime}} \mathbf{E}={ }_{\delta} \mathbf{A} \cdot \mathbf{n}
$$

The identification of each term in Eqs. (A.5) yields ${ }_{-} \mathbf{E}=\mathbf{0}$ for $S<0$ and ${ }_{+} \mathbf{E}=\mathbf{0}$ for $S>0$, corresponding to the equilibrium Eq. (A.1) inside media and + , and ${ }_{\delta} \mathbf{E}={ }_{\delta^{\prime}} \mathbf{E}=\mathbf{0}$ on $S=0$, corresponding to the jump conditions at the interface associated with the conservative Eq. (A.1).

Following exactly the same procedure with a scalar equilibrium equation, written under the general conservative form:

$$
\frac{\partial(\rho b)}{\partial t}+\nabla \cdot(\rho b \mathbf{v}+\mathbf{b})=\rho \beta
$$


we obtain the following jump conditions: $\left[\rho b\left(\mathbf{v} \cdot \mathbf{n}-w_{n}\right)+\mathbf{b} \cdot \mathbf{n}\right]+\left(\nabla \cdot{ }_{\delta} \mathbf{b}\right) /|\nabla S|=$ 0 and ${ }_{\delta} \mathbf{b} \cdot \mathbf{n}=0$ on $S=0$ (where $b, \beta$ are scalars and $\mathbf{b}$ is a vector).

Mass conservation is obtained with $\{b, \mathbf{b}, \beta\}=\{1, \mathbf{0}, 0\}$ in Eq. (A.8), which yields $\left[\rho\left(\mathbf{v} \cdot \mathbf{n}-w_{n}\right)\right]=0$. This condition means that the mass flux ${ }_{ \pm} \rho\left(_{ \pm} \mathbf{v}\right.$. $\left.\mathbf{n}-w_{n}\right)=0$ is continuous across the interface. This condition holds for shock waves. However, for an impermeable surface (fundamental assumption of this paper), the mass flux is zero across the interface so that $w_{n}={ }_{-} \mathbf{v} \cdot \mathbf{n}={ }_{+} \mathbf{v} \cdot \mathbf{n}$ :

$$
[\mathbf{v} \cdot \mathbf{n}]_{\Sigma}=0
$$

and the previous jump conditions become for an impermeable surface:

$$
[\mathbf{A} \cdot \mathbf{n}]+\frac{1}{|\nabla S|} \nabla \cdot{ }_{\delta} \mathbf{A}=\mathbf{0},[\mathbf{b} \cdot \mathbf{n}]+\frac{1}{|\nabla S|} \nabla \cdot{ }_{\delta} \mathbf{b}=0 \text { on } \Sigma
$$

(with ${ }_{\delta} \mathbf{A} \cdot \mathbf{n}=\mathbf{0}$ and ${ }_{\delta} \mathbf{b} \cdot \mathbf{n}=0$ on $\Sigma$ ).

\section{B Operators in curvilinear coordinates}

We drop the notation convention using ref, 0 and tilde for convenience. In a curvilinear coordinate system, any second order tensor $\mathbf{A}$ and vector $\mathbf{b}$ can be written as $\mathbf{A}=A^{i j} \mathbf{g}_{i} \otimes \mathbf{g}_{j}, \mathbf{b}=b^{i} \mathbf{g}_{i}$ and the following expressions hold for gradients:

$$
\nabla \mathbf{A}=\left(A_{, k}^{i j}+\Gamma_{m k}^{i} A^{m j}+\Gamma_{m k}^{j} A^{i m}\right) \mathbf{g}_{i} \otimes \mathbf{g}_{j} \otimes \mathbf{g}^{k}, \nabla \mathbf{b}=\left(b_{, j}^{i}+\Gamma_{j k}^{i} b^{k}\right) \mathbf{g}_{i} \otimes \mathbf{g}^{j}
$$

where $\Gamma_{i j}^{k}=\mathbf{g}_{i, j} \cdot \mathbf{g}^{j}$ denotes the Christoffel symbol of the second kind, satisfying the symmetry property $\Gamma_{i j}^{k}=\Gamma_{j i}^{k}$. The divergence operators are simply obtained by contracting the last two indices, yielding:

$$
\nabla \cdot \mathbf{A}=\left(A_{, j}^{i j}+\Gamma_{m j}^{i} A^{m j}+\Gamma_{m j}^{j} A^{i m}\right) \mathbf{g}_{i}, \nabla \cdot \mathbf{b}=\left(b_{, i}^{i}+\Gamma_{i k}^{i} b^{k}\right)
$$

Other useful operators are the transpose gradient of a vector $\nabla \mathbf{b}^{T}=\left(b_{, i}^{j}+\right.$ $\left.\Gamma_{i k}^{j} b^{k}\right) \mathbf{g}^{i} \otimes \mathbf{g}_{j}$ and the gradient of a scalar $\nabla f=f_{, i} \mathbf{g}^{i}$. The second order identity tensor may be written as $\mathbf{I}=\delta_{i}^{j} \mathbf{g}^{i} \otimes \mathbf{g}_{j}$. It should be outlined that the operator $(\mathbf{b} \cdot \nabla) \Psi$, sometimes used in this paper, should be understood as $\nabla \Psi \cdot \mathbf{b}$, where $\Psi$ denote any scalar, vector or tensor field.

Let us now consider a surface of discontinuity $\Sigma$, represented by $S(\mathbf{x}, t)=0$, with an attached curvilinear coordinate system defined as in Sec. 4.2. The surface $\Sigma$ is written as $\xi^{3}-f(t)=0$ in this new system, hence:

$$
\nabla S=\nabla \xi^{3}=\xi_{, i}^{3} \mathbf{g}^{i}=\mathbf{g}^{3}, \partial S / \partial t=-f^{\prime}(t)
$$


so that $f^{\prime}(t)=w_{n}|\nabla S|=w^{3}$ (where $w_{n}$ is the surface normal velocity). For any variable $\Psi$, the following geometric compatibility condition holds for the jump on $\Sigma$ :

$$
\left([\Psi]_{\xi^{3}=f(t)}\right)_{, \alpha}=\left[\Psi_{, \alpha}\right]_{\xi^{3}=f(t)}
$$

as well as the kinematic compatibility condition on $\Sigma$ :

$$
\partial / \partial t\left([\Psi]_{\xi^{3}=f(t)}\right)=[\partial \Psi / \partial t]_{\xi^{3}=f(t)}+w^{3}\left[\Psi_{, 3}\right]_{\xi^{3}=f(t)}
$$

However, note that $\left([\Psi]_{\xi^{3}=f(t)}\right)_{, 3}=0 \neq\left[\Psi_{, 3}\right]_{\xi^{3}=f(t)}$ in general.

The Christoffel symbol of the second kind can be calculated from the metric tensors thanks to the general formula: $\Gamma_{i j}^{k}=g^{k l}\left(g_{j l, i}+g_{i l, j}-g_{i j, l}\right) / 2$ (with no summation over indices). Because we have $g^{\alpha 3}=g_{\alpha 3}=0$ and $g^{33}=$ $1 / g_{33}$ for the coordinate system attached to the interface, the following useful relationships hold:

$$
\Gamma_{3 i}^{3}=g_{33, i} / 2 g_{33}=-g_{, i}^{33} / 2 g^{33}
$$

and:

$$
g^{33} \Gamma_{3 \alpha}^{\gamma}+g^{\gamma \beta} \Gamma_{\beta \alpha}^{3}=0
$$

\section{References}

[1] J. Horacek, I. Zolotarev, Acoustic-structural coupling of vibrating cylindricalshells with flowing fluid, Journal of Fluids and Structures 5 (1991) 487-501.

[2] L. Coquart, C. Depeursinge, A. Curnier, R. Ohayon, A fluid-structure interaction problem in biomechanics - prestressed vibrations of the eye by the finite-element method, Journal of Biomechanics 25 (1992) 1105-1118.

[3] A. Norris, B. Sinha, The speed of a wave along a fluid/solid interface in the presence of anisotropy and prestress, Journal of the Acoustical Society of America 25 (1995) 1147-1154.

[4] A. Degtyar, S. Rokhlin, Stress effect on boundary conditions and elastic wave propagation through an interface between anisotropic media, Journal of the Acoustical Society of America 104 (1998) 1992-2003.

[5] M. Amabili, R. Garziera, Vibrations of circular cylindrical shells with nonuniform constraints, elastic bed and added mass. part ii: Shells containing or immersed in axial flow, Journal of Fluids and Structures 16 (2002) 31-51.

[6] M. Amabili, R. Garziera, Vibrations of circular cylindrical shells with nonuniform constraints, elastic bed and added mass. part iii: Steady viscous effects on shells conveying fluid, Journal of Fluids and Structures 16 (2002) 795-809.

[7] J. Schotté, R. Ohayon, Effect of gravity on a free-free elastic tank partially filled with incompressible liquid, Journal of Fluids and Structures 18 (2003) 215-226. 
[8] M. Ottenio, M. Destrade, R. Ogden, Acoustic waves at the interface of a prestressed incompressible elastic solid and a viscous fluid, International Journal of Non-Linear Mechanics 42 (2007) 310-320.

[9] M. Fernandez, M. Moubachir, Sensitivity analysis for an incompressible aeroelastic system, Mathematical Models \& Methods in Applied Sciences 12 (2002) 1109-1130.

[10] M. Fernandez, M. Moubachir, A newton method using exact jacobians for solving fluid-structure coupling, Computers \& Structures 83 (2005) 127-142.

[11] A. Nayfeh, J. Kaiser, D. Telionis, Acoustics of aircraft engine-duct systems, AIAA Journal 13 (1975) 130-153.

[12] M. Myers, On the acoustic boundary-condition in the presence of flow, Journal of Sound and Vibration 71 (1980) 429-434.

[13] F. Farassat, M. Dunn, A simple derivation of the acoustic boundary condition in the presence of flow, Journal of Sound and Vibration 224 (1999) 384-386.

[14] B. Poirée, Les équations de l'acoustique linéaire et non-linéaire dans les fluides en mouvement, Ph.D. thesis, Thèse d'état, Université Pierre et Marie Curie, Paris VI (1982).

[15] B. Poirée, The linear and non-linear equations in the flow of an ideal fluid, Acustica 57 (1985) 5-25.

[16] O. Godin, On small amplitude waves in an inhomogeneous moving medium, Doklady Akademii Nauk 351 (1996) 322-325.

[17] O. Godin, Reciprocity and energy theorems for waves in a compressible inhomogeneous moving fluid, Wave Motion 25 (1997) 143-167.

[18] E. Goy, Résolution par une méthode d'éléments finis d'un problème vibroacoustique en présence d'un écoulement non-uniforme, Ph.D. thesis, Université de Technologie de Compiègne (2000).

[19] J. Schotté, R. Ohayon, Incompressible hydroelastic vibrations: finite element modeling of the elastogravity operator, Computers \& Structures 83 (2005) 209219.

[20] A. Norris, B. Sinha, S. Kostek, Acoustoelasticity of solid fluid composite systems, Geophysical Journal International 118 (1994) 439-446.

[21] F. Farassat, Discontinuities in aerodynamics and aeroacoustics - concept and applications of generalized derivatives, Journal of Sound and Vibration 55 (1977) 165-193.

[22] F. Farassat, Introduction to generalized functions with applications in aerodynamics and aeroacoustics, Tech. rep., NASA Technical Paper 3428 (1994), corrected copy (April 1996) (1994).

[23] R. Kanwal, Generalized Functions Theory and Technique, 2nd Edition, Birkhauser, Boston, 1998. 
[24] L. Zubov, Theory of small deformations of prestressed thin shells, Journal of Applied Mathematics and Mechanics 40 (1976) 73-82.

[25] K. Washizu, Variational Methods in Elasticity and Plasticity, 3rd Edition, Pergamon Press, 1982.

[26] B. Kim, S. Youn, A viscoelastic constitutive model of rubber under small oscillatory load superimposed on large static deformation, Archive of Applied Mechanics 71 (2001) 748-763.

[27] K.-J. Bathe, Finite Element Procedures, Prentice-Hall, New Jersey, 1996.

[28] Y.-B. Yang, S.-R. Kuo, Nonlinear Framed Structures, Prentice-Hall, Singapore, 1994.

[29] Y. Pao, W. Sachse, H. Fukuoka, Acoustoelasticity and ultrasonic measurements of residual-stresses, Physical Acoustics 17 (1984) 61-143.

[30] H. Galbrun, Propagation d'une Onde Sonore dans l'Atmosphère et Théorie des Zones de Silence, Gauthier-Villars, Paris, 1931.

[31] C. Peyret, G. Elias, Finite-element method to study harmonic aeroacoustics problems, Journal of the Acoustical Society of America 110 (2001) 661-668.

[32] F. Treyssède, G. Gabard, M. B. Tahar, A mixed finite element method for acoustic wave propagation in moving fluids based on an eulerian-lagrangian description, Journal of the Acoustical Society of America 113 (2003) 705-716.

[33] K. Hutter, K. Johnk, Continuum Methods of Physical Modeling, SpringerVerlag, Berlin, 2004.

[34] J. Coirier, Mécanique des Milieux Continus, Dunod, Paris, 1997.

[35] B. Poirée, Les équations de l'acoustique linéaire dans un fluide parfait au repos à caractéristiques indéfiniment différentiables par morceaux, Revue du Cethedec 52 (1977) 69-79.

[36] A. Gray, E. Abbena, S. Salamon, Modern Differential Geometry of Curves and Surfaces with Mathematica, 3rd Edition, Chapman \& Hall, Boca Raton, 2006.

[37] J.-L. Synge, A. Schild, Tensor Calculus, Dover, 1978.

[38] D. Chapelle, K. J. Bathe, The Finite Element Analysis of Shells - Fundamentals, Springer, 2003.

[39] S. Horowitz, R. Sigman, B. Zinn, An iterative finite element-integral technique for predicting sound radiation from turbofan inlets in steady flight, AIAA Journal 24 (1986) 1256-1262.

[40] W. Eversman, D. Okunbor, Aft fan duct acoustic radiation, Journal of Sound and Vibration 213 (1998) 235-257.

[41] W. Eversman, The boundary condition at an impedance wall in a non-uniform duct with potential mean flow, Journal of Sound and Vibration 246 (2001) $63-69$. 
[42] W. Eversman, The boundary condition at an impedance wall in a non-uniform duct with potential mean flow (erratum), Journal of Sound and Vibration 258 (2002) 791-792.

[43] S. Rienstra, W. Eversman, A numerical comparison between the multiple-scales and finite-element solution for sound propagation in lined flow ducts, Journal of Fluid Mechanics 437 (2001) 367-384.

[44] F. Treyssède, M. B. Tahar, Validation of a finite element method for sound propagation and vibro-acoustic problems with swirling flows, Acta Acustica United with Acustica 90 (2004) 731-745.

[45] G. Gabard, F. Treyssède, M. B. Tahar, A numerical method for vibro-acoustic problems with sheared mean flows, Journal of Sound and Vibration 272 (2004) 991-1011. 